\title{
Validación y selección de indicadores para la evaluación de competencias. Aplicación a la competencia transversal de creatividad, innovación y emprendimiento
}

\author{
Prats-Montalbán, J.M. b, Ruiz L. a, Boza A. ${ }^{\text {a, }}$ Fernández-Diego M. a , Gordo M.L. a , \\ Alarcón F. b, Alemany M.M.E. ${ }^{\text {b }}$, y Cuenca L. ${ }^{\text {a }}$ \\ ${ }^{a}$ Escuela Técnica Superior de Ingeniería Informática. Universitat Politécnica de \\ Valencia (Iruiz@omp.upv.es, aboza@omp.upv.es, marferdi@omp.upv.es, \\ magormon@upvnet.upv.es, Ilcuenca@omp.upv.es), ${ }^{b}$ Escuela Técnica Superior de \\ Ingeniería Industrial. Universitat Politécnica de Valencia (jopramon@eio.upv.es, \\ faualva@omp.upv.es, mareva@omp.upv.es )
}

\begin{abstract}
The study focuses on establishing a methodology for the evaluation and selection of objective indicators based on statistical evidence, in order to determine how the scope of a given competence should be evaluated and the indicators that must be used to do so. By means of multivariate statistical models, prediction models of the mark of the competence are established from the marks of each of the indicators, as well as the degree of internal relationship between the indicators. In this way, it is possible to select those indicators that are providing information as independently as possible to that provided by any other indicator. This is a great help for the achievement of two main objectives: 1) to homogenize the items to be evaluated by each and every one of the teachers when evaluating the competence of interest; 2) increase the level of objectivity of the evaluation
\end{abstract}

Keywords: transversal competence, variable selection, validation, multivariate models, competence evaluation.

\footnotetext{
Resumen

El estudio se centra en establecer una metodología de evaluación y selección de indicadores objetiva basada en la evidencia estadística, con el fin de determinar cómo ha de evaluarse el alcance de una determinada competencia y los indicadores que hay que utilizar para ello. Por medio de modelos estadísticos multivariantes se establecen modelos de predicción de la nota de la competencia a partir de las notas de cada uno de los indicadores, así como el grado de relación interna entre los indicadores. De esta manera, es posible seleccionar aquellos indicadores que estén aportando información lo más independiente posible a aquella proporcionada por cualquier otro indicador. Esto resulta de gran ayuda para la consecución de dos objetivos principales: 1) homogeneizar los ítems a evaluar por todos y cada uno de los profesores a la hora de evaluar la competencia de interés; 2) incrementar el nivel de objetividad de la evaluación ; 3) simplificar el proceso de evaluación reduciendo el número de indicadores a los representativos.
} 
Palabras clave: competencias transversales, selección de indicadores, validación, modelos multivariantes.

\section{Introducción}

En la actualidad, la determinación (selección) de los indicadores se realiza normalmente a partir de reuniones de grupos expertos que seleccionan, entre un grupo extenso de potenciales indicadores, cuáles son los que tienen una relación clara con la competencia; estableciendo a continuación un baremo (que puede variar en función del profesor) a partir del cual se determina la nota alcanzada en la competencia evaluada.

Sin embargo, esta determinación por grupos expertos presenta una serie de problemas. Por un lado, no es posible garantizar que la selección de indicadores llevada a cabo por diferentes grupos expertos sea la misma, debido a la naturaleza intrínsecamente subjetiva (basada en el conocimiento y experiencia de cada grupo de expertos). Por otro, el establecimiento a priori de un baremo tiene como consecuencia que, cualquier estudio a posteriori de la significación estadística de la relación del indicador con la competencia de interés, se encuentre necesariamente afectada por el coeficiente utilizado en el baremo a la hora de ponderar la nota de la competencia como combinación lineal de las notas obtenidas en los indicadores utilizados.

En definitiva, es importante establecer el baremo de manera objetiva y validar que los indicadores asociados miden realmente lo que se desea medir, dando así validez a los resultados (Messick, 1996). Existen dos maneras de abordar los procesos de validación: por una parte, sobre las características del propio método de evaluación y, por otra, sobre la interpretación de los resultados (Borsboom, Mellenbergh, \& van Heerden, 2004; Brown et al., 1997). En el contexto que nos ocupa, la validación se realiza sobre el propio método de evaluación, respondiendo a la pregunta ¿los indicadores asociados a la rúbrica para la competencia de creatividad, innovación y emprendimiento han sido escogidos de manera objetiva y precisa?

La respuesta a esta pregunta hace necesario establecer una metodología de evaluación y selección de indicadores objetiva y basada en la evidencia estadística. Dicha metodología comienza con una recogida de datos, para todos y cada una de los indicadores incluidos en la batería inicial; que deben evaluarse de manera independiente a la evaluación de la competencia de interés. Sólo así es posible validar la significación estadística de la relación entre el indicador y la competencia. Una vez recogidos los datos correctamente, se hace necesario recurrir a modelos estadísticos multivariantes capaces, no sólo de crear modelos de predicción (de la nota de la competencia a partir de las notas de cada uno de los indicadores), sino también de establecer el grado de relación interna entre los indicadores. De esta manera, es posible seleccionar aquellos indicadores que estén aportando claramente información lo más independiente posible a aquella proporcionada por cualquier otro indicador incluido en el estudio. 
Asimismo, el recurso a estos modelos permite, por medio de técnicas de selección de variables determinar cuáles son los indicadores que presentan una clara significación estadística en el modelo (i.e. su relación con la nota de la competencia no es fruto del azar); proporcionando además, tras su selección, un baremo objetivo que permite predecir, una vez puesto en marcha el procedimiento, la nota de la competencia por parte de todo el profesorado a partir de un grupo reducido de indicadores seleccionado de manera objetiva en base a criterios científicos; objetivo final del presente proyecto.

\section{Objetivos}

El objetivo principal del estudio es establecer un procedimiento objetivo y basado en el método científico para la selección de indicadores asociados a la competencia de creatividad, innovación y emprendimiento, del cual se derivan los siguientes objetivos secundarios (Prats et al., 2016):

1. Definir una metodología para evaluar las rúbricas relacionadas con una determinada competencia, que se podría generalizar al total de competencias transversales de la UPV.

2. Validar y seleccionar un conjunto reducido de indicadores estadísticamente significativos, mediante la aplicación de modelos estadísticos multivariantes, que tengan una elevada capacidad predictiva del valor global de la competencia analizada.

3. Obtener, a partir de estos modelos, los coeficientes asociados a cada indicador, según el peso que cada uno tenga en la evaluación de la competencia de interés.

\section{Contextualización de la experiencia}

El estudio se ha aplicado sobre tres asignaturas de la ETSI Informática y tres de la ETSI Industrial. Las actividades definidas pueden ser transferibles a otras asignaturas y otros centros de la UPV. De esta manera, el proyecto ha involucrado a cuatro titulaciones, ocho profesores, seis asignaturas y alrededor de 300-400 alumnos. Las asignaturas involucradas, correspondientes a las titulaciones y cursos, son las que se indican a continuación.

\section{- Grado de Ingeniería Informática (E.T.S.I. Informática)}

o Fundamentos de Organización de Empresas

- Obligatoria

- Número de estudiantes: 40

- Curso: $1^{\circ}$

o Modelos de Negocio y Áreas Funcionales de la Organización

- Optativa

- Número de estudiantes: 55

- Curso: $4^{\circ}$

- Máster Universitario de Ingeniería Informática (E.T.S.I. Informática)

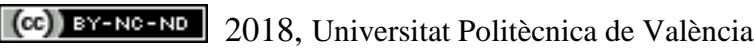

Congreso In-Red (2018) 
o Gestión y Gobierno de las TI

- Optativa

- $\quad$ Número de estudiantes: 35

- Curso $1^{\circ}$

- Grado en Ingeniería de Organización E.T.S.I. Industrial

o Métodos cuantitativos de Organización Industrial

- Obligatoria

- Número de estudiantes: 80

- Curso: $3^{\circ}$

o Gestión por Procesos de negocio. Implantación, Desarrollo y Simulación

- Optativa

- $\quad$ Número de estudiantes: 35

- Curso: $4^{\circ}$

- Grado en Ingeniería Química E.T.S.I. Industrial

o Estadística

- Obligatoria

- Número de estudiantes: 100

- Curso: $1^{\circ}$

\section{Desarrollo de la innovación}

El desarrollo del estudio fue complejo debido a diferentes complicaciones que fueron surgiendo conforme se pretendía avanzar en el mismo y que se detallan a continuación:

- Existencia de una gran cantidad de potenciales indicadores a evaluar por medio de diferentes actividades, y la dificultad asociada a la selección óptima de los mismos.

- $\quad$ Aparición de diferentes indicadores en función de la asignatura.

- Dudas acerca de la utilidad de las rúbricas a la hora de evaluar los indicadores.

- Dificultad en la evaluación de los indicadores, necesidad de determinar las actividades a realizar.

- $\quad$ Limitaciones en la cantidad de indicadores a evaluar debido a falta de recursos.

El estudio, teniendo en cuenta estas limitaciones, comenzó con la revisión y análisis de las actividades existentes en la bibliografía relacionadas con la innovación, creatividad y emprendimiento que pudieran servir de base para la adquisición de la competencia y pudieran ser adaptadas a las asignaturas.

A partir de esta revisión, se realizó una selección de una batería de 24 indicadores a partir de la fuentes bibliográficas y consultas con el Instituto de Ciencias de la Educación (ICE) de la UPV, dada la limitación en los recursos disponibles. Hay que resaltar que idealmente habría que haber utilizado todos los indicadores existentes, con el fin de que los modelos sean los que lleven a cabo la selección de manera objetiva, basada en datos. El hecho de no disponer de recursos suficientes limitó la cantidad de indicadores a utilizar en el estudio. En cualquier caso, este hecho no limita la utilidad del trabajo, el cual se centra en el 
establecimiento de una metodología objetiva para la selección de indicadores, independientemente de que se disponga o no de la totalidad desde un principio. Conocidos los indicadores, se diseñaron las actividades adecuadas para poder evaluarlos posteriormente, y se evaluaron por parte del profesorado.

La lista de indicadores seleccionados ha sido la que se indica a continuación (extraída de Boza et al., 2014, Fernández-Diego et al, 2014, Cuenca et al., 2015a, Cuenca et al., 2015b, Cuenca et al., 2016):

1. ' 'Analizar una situación dada e identificar aspectos de mejora'

2. 'Aportar ideas originales en cuanto a contenido'

3. 'Aportar ideas originales en cuanto a la forma de materializarse'

4. 'Aportar sugerencias a las ideas, situaciones, casos o problemas que se plantean'

5. 'Aportar sugerencias propias de calidad ante situaciones o problemas que se presentan'

6. 'Buscar nuevos procedimientos y métodos para hacer las cosas'

7. 'Buscar y proponer nuevos métodos y soluciones ante situaciones problemáticas reales o hipotéticas'

8. 'Disposición a actuar en contra de la tradición si esta impide mejoras en el rendimiento'

9. 'Encontrar nuevos métodos y procesos para hacer las cosas'

10. 'Expresar a otro las nuevas ideas que se han generado'

11. 'Familiarizarse con los instrumentos y técnicas de generación de ideas'

12. 'Generar ideas originales y de calidad, que se pueden plasmar de una manera formal y defenderlas en situaciones conocidas y desconocidas'

13. 'Generar y transmitir nuevas ideas o generar alternativas innovadoras a las situaciones o problemas conocidos que se plantean'

14. 'Integrar conocimientos de varias disciplinas, fuentes o ámbitos para generar ideas novedosas a situaciones conocidas o desconocidas'

15. 'Introducir nuevos procedimientos y acciones en el propio proceso de trabajo para responder mejor a las limitaciones y problemas detectados'

16. 'Plasmar de manera formal las ideas que se han generado'

17. 'Proponer ideas y soluciones innovadoras tanto en cuanto al contenido, como al proceso para llevarlas a cabo'

18. 'Proponer sugerencias y alternativas adecuadas a las necesidades de la tarea'

19. 'Reflexionar sobre nuevas formas de hacer las cosas'

20. 'Responder con detalle e integridad de respuestas'

21. 'Responder con flexibilidad (variabilidad de ideas en las respuestas)'

22. 'Responder con fluidez. Número de respuestas contestadas (fluidez)'

23. 'Responder con originalidad (rareza de la respuesta)'

24. 'Transmitir sus ideas con coherencia y eficacia' 


\section{Metodología y técnicas utilizadas para el tratamiento y análisis de los datos.}

La propia naturaleza del trabajo consiste en la creación de una metodología de recogida y análisis de datos. Dicha metodología ha consistido en la aplicación de un método de selección de variables combinado con la utilización de modelos estadísticos de proyección sobre estructuras latentes de Regresión sobre Mínimos Cuadrados Parciales, PLS (Geladi and Kowalski, 1986), a partir de datos recogidos en un proceso de evaluación por pares de actividades. Tal como explica el profesor Romero (Romero y Zúnica, 2013), "La Estadística es la ciencia cuyo objeto es la obtención y el análisis de datos mediante el recurso a modelos matemáticos y a herramientas informáticas". Por ello, si se desea obtener información a partir de los datos, éstos deben obtenerse de manera correcta. Dicho de otra manera, es improbable obtener conclusiones correctas a partir de datos obtenidos de manera incorrecta por lo que se ha puesto mucha atención en la recolección de datos, tal y como se detalla/explica a continuación.

\subsection{Recogida de los datos}

En base a los 24 indicadores seleccionados y a las actividades desarrolladas, se evaluaron por separado el grado de competencia global a partir de la actividad, y a posteriori el nivel alcanzado en cada uno de los indicadores; todos ellos de 0 a 10, por ser una escala sobre la cual (con la que) los profesores están bien entrenados a la hora de puntuar. Esta metodología garantiza que no se establece un baremo, ni interno (psicológico) ni explícito (mediante un baremo), a la hora de relacionar la nota final de la competencia con las notas asignadas a cada uno de los indicadores. Hay que tener en cuenta que el objetivo final es establecer una metodología objetiva de selección de indicadores para un conjunto general, del cual el (sub)conjunto actual no es más que una selección; a la vez que permitir que la metodología propuesta sea exportable al total de competencias.

En el momento de la realización del presente trabajo, sólo fue posible disponer de un total de 80 evaluaciones, por lo que la obtención de un baremo objetivo, se ha tenido que dejar fuera del estudio.

\subsection{Análisis de los datos}

El análisis de los datos se ha realizado a través de modelos estadísticos multivariantes de proyección sobre estructuras latentes, con el fin de manejar de manera eficiente variables (indicadores) que probablemente van a mostrar un elevado grado de correlación. Dado que se dispone de una estructura de predictores (indicadores) a partir de los cuales se pretende predecir el valor final de una variable de salida (el grado de competencia), es necesario recurrir a modelos de predicción, como es el caso de, entre otros, los modelos PLS (Geladi and Kowalski, 1986).

El objetivo del modelo PLS es explicar la relación entre dos conjuntos de variables $\mathbf{X}$ e $\mathbf{Y}$, así como la variabilidad tanto en $\mathbf{X}$ como en $\mathbf{Y}$, pero maximizando la Covarianza $(\mathbf{X}, \mathbf{Y})$. La ventaja de estos modelos estadísticos multivariantes de proyección sobre estructuras 
latentes frente a modelos típicos, tales como el de Regresión Líneal Múltiple, es el manejo de estructuras de datos con múltiples variables correlacionadas, que comprimen de manera eficiente, eliminando los problemas de invertibilidad de la matriz de varianzas-covarianzas que pueden dar lugar a estimaciones inestables de los coeficientes, y por tanto malas predicciones y/o malas selección de variables (indicadores en nuestro caso).

A partir de la utilización de modelos PLS, se ha llevado a cabo la selección de indicadores mediante la integración de un proceso de selección basado en distribuciones libres, en una secuencia de doble validación cruzada (Quintás et al., 2012). Dicha técnica consiste en los siguientes pasos:

1. A partir de una selección aleatoria del conjunto global de datos, creación de los conjuntos de Training, Validation, y Test.

2. Construcción del modelo a partir del conjunto de Training, con una variable latente.

3. Determinación del poder de predicción con el Validation set.

4. Creación de las distribuciones libres, a partir de la destrucción de la variable a predecir (nota de la competencia por parte de los profesores).

5. Eliminación de las variables no significativas del training set.

6. Volver al paso 2, hasta que no se mejore el paso 3.

7. Obtener el valor real de predicción a partir del Test set.

8. Repetir los pasos 1-7 un elevado número de veces.

9. Elegir las variables que más veces se han repetido para un determinado nivel de predicción mínimo, sobre el Test set.

\section{Resultados}

Finalmente, los indicadores elegidos de la lista analizada, a partir de la metodología propuesta, han sido:

- 'Aportar sugerencias propias de calidad ante situaciones o problemas que se presentan'

- 'Introducir nuevos procedimientos y acciones en el propio proceso de trabajo para responder mejor a las limitaciones y problemas detectados'

- 'Reflexionar sobre nuevas formas de hacer las cosas'

- 'Responder con originalidad (rareza de la respuesta)'

Como se ha comentado anteriormente, no se ha obtenido el coeficiente a aplicar a cada uno de estos indicadores, ya que se considera conveniente para ello disponer de un tamaño mayor de muestra.

Para comparar el resultado obtenido con los indicadores que actualmente se están utilizando en la rúbrica de evaluación, debemos tener en cuenta que la actividad llevada a cabo en el aula ha sido la 6-3-5 (Rohbarch, 1969; Cuenca et al., 2016b). Está actividad está más indicada para los aspectos relacionados con la creatividad y con plasmar las ideas que 
surgen (brainwriting) que en llevar a cabo un plan de acción y analizar el valor de la innovación propuesta.

También hay que tener en cuenta que los indicadores resultado de la investigación no están clasificados por niveles (N1: primer y segundo curso, N2: tercer y cuarto curso y N3: máster).

A partir de lo anterior se puede establecer una equivalencia limitada entre los indicadores inicialmente propuestos y los que ha revelado la investigación, tal y como se indica en la Tabla 1.

Tabla 1. Equivalencia entre los indicadores seleccionados por el método propuestos y los actualmente utilizados.

\begin{tabular}{|c|c|c|}
\hline $\begin{array}{c}\text { Fases del proceso } \\
\text { creatividad, } \\
\text { innovación y } \\
\text { emprendimiento que } \\
\text { se abordan en la } \\
\text { actividad } 6-3-5\end{array}$ & Indicadores previamente definidos & $\begin{array}{c}\text { Indicadores seleccionados tras la } \\
\text { investigación }\end{array}$ \\
\hline $\begin{array}{c}\text { Identificar } \\
\text { oportunidad }\end{array}$ & $\begin{array}{l}\text { N1:Se cuestiona la realidad } \\
\text { N2:Identifica oportunidades y/o } \\
\text { aspectos de mejora } \\
\text { N3:Integra conocimientos de otras } \\
\text { disciplinas }\end{array}$ & $\begin{array}{l}\text { 'Reflexionar sobre nuevas formas de } \\
\text { hacer las cosas' }\end{array}$ \\
\hline Generar Ideas & $\begin{array}{l}\text { N1:Aporta ideas } \\
\text { N2:Aporta ideas y planteamientos } \\
\text { originales } \\
\text { N3: Adopta enfoques creativos en el } \\
\text { contenido y modo de realización }\end{array}$ & $\begin{array}{l}\text { 'Aportar sugerencias propias de } \\
\text { calidad ante situaciones o problemas } \\
\text { que se presentan' } \\
\text { 'Responder con originalidad (rareza } \\
\text { de la respuesta)' }\end{array}$ \\
\hline Ejecución & $\begin{array}{l}\text { N1:Plasma de manera formal las } \\
\text { ideas } \\
\text { N2:Emplea estrategias y/o técnicas } \\
\text { creativas para } \\
\text { plasmar de manera formal las ideas } \\
\text { y soluciones } \\
\text { N3:Propone un plan de acción }\end{array}$ & $\begin{array}{l}\text { 'Introducir nuevos procedimientos y } \\
\text { acciones en el propio proceso de } \\
\text { trabajo para responder mejor a las } \\
\text { limitaciones y problemas detectados' }\end{array}$ \\
\hline
\end{tabular}

\section{Conclusiones y proyección de futuro}

El presente trabajo ha permitido validar una metodología previamente estudiada en el campo de la Estadística multivariante, dentro de otro campo con valoraciones de carácter subjetivo, lo cual aporta un grado de dificultad añadido. Los resultados son muy prometedores, si bien el presente trabajo se presenta como una primera aproximación a este tipo de datos. 
Como trabajo futuro, queda ampliar el tamaño de muestra, con el fin de validar los indicadores seleccionados en el presente estudio; así como el establecimiento de un baremo objetivo a partir de los indicadores que finalmente sean elegidos.

Uno de los aspectos clave del presente estudio es su capacidad de universalización mediante reuniones del grupo de trabajo (y de otros grupos expertos en otra competencias) con el ICE y/o Vicerrectorado. Se podría analizar la posibilidad de exportarla, con carácter general, a toda la relación de competencias transversales definidas por la UPV.

\section{Referencias}

BORSBOOM, D.; MELLENBERGH, G. J.; VAN HEERDEN, J. (2004). "The concept of validity”, en Psychological Review , vol. 111, pp. 1061-1071.

BOZA. A.; CUENCA, L.; FERNÁNDEZ-DIEGO, M.; RUIZ, L.; GORDO, M.L.; ALARCÓN, F.; ALEMANY, M.M.E.; POLER, R. (2014). "Innovation, creativity and entrepreneurship competence in higher education. Learning objectives and measurement", en ICERI2014 Proceedings, pp. 405-411.

BROWN, G.; BULL, J.; PENDLEBURY, M. (1997). Assessing student learning in higher education. London: Routledge.

CUENCA L., FAUSTINO ALARCÓNB, ANDRÉS BOZAC , MARTA FERNÁNDEZDIEGOD, LEONOR RUIZ, MARI LUZ GORDO, RAUL POLER, MAREVA ALEMANY (2015a) "Rúbrica para la Evaluación de la Competencia Innovación, Creatividad y Emprendimiento en máster”, en Congreso In-Red 2015 Universitat Politècnica de València. DOI: http://dx.doi.org/10.4995/INRED2015.2015.1610

CUENCA L., FERNÁNDEZ-DIEGO M., GORDO M., RUIZ L., ALEMANY M.M.E., ORTIZ A. (2015B) MEASURING COMPETENCIES IN HIGHER EDUCATION. "The Case of Innovation Competence”, en Peris-Ortiz M., Merigó Lindahl J. (eds) Sustainable Learning in Higher Education. Innovation, Technology, and Knowledge Management. Springer, pp. 131-141

CUENCA, L., ALARCÓN, F., BOZA, A., ET AL., (2016a). "Rubric to assess the competence of innovation, creativity and enterpreneurship in bachelor degree" en Brazilian Journal of Operations \& Production Management, vol 13(1), pp.118-123.

CUENCA, L.; ALARCÓN, F.; ALEMANY, MME.; BOZA, A.; FERNÁNDEZ DIEGO, M.; GORDO, ML.; RUIZ, L. (2016b). "La técnica 6-3-5 extendida, para la competencia de creatividad, innovación y emprendimiento”. http://hdl.handle.net/10251/68332.

FERNÁNDEZ-DIEGO M., RUIZ L., GORDO M.L., M.M.E. ALEMANY, BOZA A., CUENCA L. (2014) "Objetivos de aprendizaje para grado y máster de la competencia genérica de innovación, creatividad y emprendimiento”, en Jornadas In-Red 2014 pp.1-9

GELADI, P.; KOWALSKI, B.R. (1986) “Partial Least-Squares Regression: A Tutorial”, en Analytica Chimica Acta, vol. 185, pp. 1-17.

2018, Universitat Politècnica de València 
MESSICK, S. (1996). "Validity of performance assessments", en G. Phillips (Ed.), Technical issues in large-scale performance assessment, pp. 1-18.

QUINTÁS, G.; PORTILLO, N.; GARCÍA-CAÑAVERAS, J.C.; CASTELL, J.V.; FERRER, A.; LAHOZ, A. (2012) "Chemometric approaches to improve PLSDA model outcome for predicting human non-alcoholic fatty liver disease using UPLC-MS as a metabolic profiling tool”, en Metabolomics , vol. 8, pp. 86-98.

PRATS-MONTALBÁN J.M., F. ALARCÓN, M.M.E. ALEMANY, A. BOZA, M.L. GORDO, M. FERNÁNDEZ-DIEGO, L. RUIZ, L. CUENCA (2016) “Innovation project to validate and select items for assessing transversal competencies in higher education”, en ICERI2016 Proceedings, pp. 61-68.

ROHRBACH, BERND (1969) "Kreativ nach Regeln - Methode 635, eine neue Technik zum Lösen von Problemen". Creativo mediante reglas - Método 635, una nueva técnica para resolver problemas, en Absatzwirtschaft, vol. 12, pp. 73-75.

ROMERO VILLAFRANCA, R., ZÚNICA RAMAJO, L. (2013) Métodos estadísticos para ingenieros. Valencia: Universidad Politécnica de Valencia (Ref.: 4) 